Z. Wei and C. Pang

Nagoya Math. J.

Vol. 162 (2001), 127-148

\title{
POSITIVE SOLUTIONS OF NONRESONANT SINGULAR BOUNDARY VALUE PROBLEM OF SECOND ORDER DIFFERENTIAL EQUATIONS
}

\author{
ZHONGLI WEI ${ }^{1}$ AND CHANGCI PANG
}

\begin{abstract}
This paper investigates the existence of positive solutions of nonresonant singular boundary value problem of second order differential equations. A necessary and sufficient condition for the existence of $C[0,1]$ positive solutions as well as $C^{1}[0,1]$ positive solutions is given by means of the method of lower and upper solutions with the fixed point theorems.
\end{abstract}

\section{$\S 1$. Introduction}

The theory of singular boundary value problems has become an important area of investigation in recent years (see [1-7] and the references therein). Consider the singular boundary value problems of second order ordinary differential equation

$$
\left\{\begin{array}{l}
-x^{\prime \prime}+\rho p(t) x=f(t, x), \quad t \in(0,1), \\
a x(0)-b x^{\prime}(0)=0, \quad c x(1)+d x^{\prime}(1)=0,
\end{array}\right.
$$

where $\rho>0$ is such that

$$
\begin{cases}-x^{\prime \prime}+\rho p(t) x=0, & t \in(0,1) \\ a x(0)-b x^{\prime}(0)=0, & c x(1)+d x^{\prime}(1)=0\end{cases}
$$

has only the trivial solution, and where $p(t) \in C(0,1), p(t) \geq 0, t \in(0,1)$, $a \geq 0, b \geq 0, c \geq 0, d \geq 0, a+b>0, c+d>0, \delta=a c+a d+b c>0$. For convenience, we list the following hypothesis.

$$
\int_{0}^{1} t(1-t) p(t) d t<\infty ; \quad \text { also }
$$

Received May 24, 1999.

1991 Mathematics Subject Classification: 34B15.

${ }^{1}$ Research supported by NNSF-China (10071043) and NSF of Shandong Province (Y2000A06) 


$$
\begin{gathered}
\lim _{t \rightarrow 0^{+}} t^{2} p(t)=0 \text { if } \int_{0}^{1}(1-t) p(t) d t=\infty ; \quad \text { and } \\
\lim _{t \rightarrow 1^{-}}(1-t)^{2} p(t)=0 \text { if } \int_{0}^{1} t p(t) d t=\infty
\end{gathered}
$$

$$
\begin{gathered}
\int_{0}^{1} t p(t) d t<\infty ; \quad \text { also } \\
\lim _{t \rightarrow 0^{+}} t^{2} p(t)=0 \text { if } \int_{0}^{1} p(t) d t=\infty
\end{gathered}
$$

$$
\begin{gathered}
\int_{0}^{1}(1-t) p(t) d t<\infty ; \quad \text { also } \\
\lim _{t \rightarrow 1^{-}}(1-t)^{2} p(t)=0 \text { if } \int_{0}^{1} p(t) d t=\infty
\end{gathered}
$$

$\left(\mathrm{H}_{5}\right) \quad f(t, x) \in C((0,1) \times(0,+\infty),[0,+\infty)), f(t, 1) \not \equiv 0$ for $t \in(0,1)$, and there exist constants $\lambda, \mu, N, M(-\infty<\lambda<0<\mu<1,0<N \leq 1 \leq M)$, such that, for $t \in(0,1)$ and $x \in(0,+\infty)$,

$$
\begin{gathered}
\ell^{\mu} f(t, x) \leq f(t, \ell x) \leq \ell^{\lambda} f(t, x) \quad \text { if } \quad 0 \leq \ell \leq N \\
\ell^{\lambda} f(t, x) \leq f(t, \ell x) \leq \ell^{\mu} f(t, x) \quad \text { if } \ell \geq M
\end{gathered}
$$

Typical functions that satisfy the above sublinear hypothesis are those taking the form

$$
f(t, x)=\sum_{k=1}^{n} p_{k}(t) x^{\lambda_{k}} ;
$$

here $p_{k}(t) \in C(0,1), p_{k}(t)>0$ on $(0,1), \lambda_{k}<1, k=1,2, \ldots, n$. 
By singularity we mean that the functions $p, f$ in (1.1) are allowed to be unbounded at the end points $t=0$ and $t=1$. A function $x(t) \in$ $C[0,1] \cap C^{2}(0,1)$ is called a $C[0,1]$ (positive) solution of (1.1) if it satisfies (1.1) $(x(t)>0$ for $t \in(0,1))$. A $C[0,1]$ (positive) solution of (1.1) is called a $C^{1}[0,1]$ (positive) solution if $x^{\prime}\left(0^{+}\right)$and $x^{\prime}\left(1^{-}\right)$both exist $(x(t)>0$ for $t \in(0,1))$.

In the special cases i): $b=d=0, p(t)=0, f(t, x)=p_{1}(t) x^{-\lambda_{1}}, \lambda_{1}>0$ and ii): $b=d=0, p(t)=0, f(t, x)=p_{1}(t) x^{\lambda_{1}}, 0<\lambda_{1}<1$, where $p_{1}(t) \in C(0,1), p_{1}(t)>0$ on $(0,1)$, the existence and uniqueness of positive solutions of (1.1) have been studied completely by Taliaferro in [3] with the shooting method and by Zhang in [4] with the method of lower and upper solutions, respectively. A sufficient condition for the existence of $C[0,1]$ solutions of the singular problem (1.1) in the case $b=d=0$ was given by D. O'Regan in [5] with a continuous theorem. In the special cases iii): $p(t)=$ $0, f(t, x)=p_{1}(t) x^{-\lambda_{1}}, \lambda_{1}>0$ and iv $): p(t)=0, f(t, x)=p_{1}(t) x^{\lambda_{1}}, 0<$ $\lambda_{1}<1$, where $p_{1}(t) \in C(0,1), p_{1}(t)>0$ on $(0,1)$, the existence of positive solutions of (1.1) has been studied by Wei in [6] and [7] with the method of lower and upper solutions.

Now, in this paper, we shall give a necessary and sufficient condition for the existence of $C[0,1]$ positive solutions as well as $C^{1}[0,1]$ positive solutions of the singular problem (1.1) by using the method of lower and upper solutions with the fixed point theorems, which is different from that of $[3-5]$.

\section{$\S 2$. Several lemmas}

LEMma 1. Suppose $\left(\mathrm{H}_{1}\right)$ holds.

(i) Then

$$
\left\{\begin{array}{l}
-x^{\prime \prime}+\rho p(t) x=0, \quad t \in(0,1), \\
x(0)=0, \quad x^{\prime}(0)=1
\end{array}\right.
$$

has a unique positive increasing solution $e_{1}(t) \in C[0,1] \cap C^{1}[0,1)$.

(ii) Then

$$
\left\{\begin{array}{l}
-x^{\prime \prime}+\rho p(t) x=0, \quad t \in(0,1), \\
x(1)=0, \quad x^{\prime}(1)=-1
\end{array}\right.
$$

has a unique positive decreasing solution $e_{2}(t) \in C[0,1] \cap C^{1}(0,1]$.

In addition, if $\left(\mathrm{H}_{2}\right)$ holds, then $e_{1}(t) \in C^{1}[0,1] ;$ if $\left(\mathrm{H}_{3}\right)$ holds, then $e_{2}(t) \in C^{1}[0,1]$; therefore, if $\left(\mathrm{H}_{4}\right)$ holds, then $e_{1}(t), e_{2}(t) \in C^{1}[0,1]$. 
Proof. Similar to that of Theorem 2.1 in [5], we can obtain that there exists a unique $w_{1} \in C[0,1]$ with

$$
w_{1}(t)=1+\frac{\rho}{t} \int_{0}^{t} \int_{0}^{s} \tau p(\tau) w_{1}(\tau) d \tau d s
$$

and $e_{1}(t)=t w_{1}(t) \in C[0,1] \cap C^{1}[0,1)$ is a solution of (2.1). In the following, we shall prove that $e_{1}(t)$ is a positive increasing function. In fact, if $e_{1}(t)$ is not increasing, then from $e_{1}(0)=0, e_{1}^{\prime}(0)=1$, there exist positive numbers $0<t^{*}<\eta<1$ such that $e_{1}^{\prime}\left(t^{*}\right)<0$ and $e_{1}(t)>0$ for $t \in(0, \eta)$. Therefore,

$$
\int_{0}^{t^{*}}\left(-e_{1}^{\prime \prime}(t)+\rho p(t) t w_{1}(t)\right) d t \geq-e_{1}^{\prime}\left(t^{*}\right)+1>0
$$

which contradicts

$$
-e_{1}^{\prime \prime}(t)+\rho p(t) t w_{1}(t)=0, \quad t \in(0,1)
$$

Hence, $e_{1}(t)$ is an increasing function. From $e_{1}(t)>0$ for $t \in(0, \eta)$, we have $e_{1}(t)>0$ for $t \in[0,1]$. Consequently, $w_{1}(t) \geq 0$ for $t \in[0,1]$ and $w_{1}(1)=e_{1}(1)>0$.

Similarly, we can obtain that there exists a nonnegative function $w_{2} \in$ $C[0,1]$ with

$$
w_{2}(t)=1+\frac{\rho}{1-t} \int_{t}^{1} \int_{s}^{1}(1-\tau) p(\tau) w_{2}(\tau) d \tau d s
$$

and $e_{2}(t)=(1-t) w_{2}(t) \in C[0,1] \cap C^{1}(0,1]$ is a positive decreasing solution of $(2.2)$.

Obviously, if $\left(\mathrm{H}_{2}\right)$ holds, then $e_{1}(t) \in C^{1}[0,1]$; if $\left(\mathrm{H}_{3}\right)$ holds, then $e_{2}(t) \in$ $C^{1}[0,1]$; therefore, if $\left(\mathrm{H}_{4}\right)$ holds, then $e_{1}(t), e_{2}(t) \in C^{1}[0,1]$. The proof is complete.

Remark 1. If $p(t)=0$, then $e_{1}(t)=t, e_{2}(t)=1-t, w_{1}(t)=w_{2}(t)=1$.

By Lemma 1, we can obtain Lemma 2.

Lemma 2. (i) Suppose that $\left(\mathrm{H}_{3}\right)$ holds. Then

$$
u(t)=\left(a e_{2}(0)-b e_{2}^{\prime}(0)\right) e_{1}(t)+b e_{2}(t) \in C[0,1] \cap C^{1}[0,1)
$$


is a positive increasing solution of the following problem

$$
\left\{\begin{array}{l}
-x^{\prime \prime}+\rho p(t) x=0, \quad t \in(0,1), \\
a x(0)-b x^{\prime}(0)=0
\end{array}\right.
$$

(ii) Suppose that $\left(\mathrm{H}_{2}\right)$ holds. Then

$$
v(t)=d e_{1}(t)+\left(c e_{1}(1)+d e_{1}^{\prime}(1)\right) e_{2}(t) \in C[0,1] \cap C^{1}(0,1]
$$

is a positive decreasing solution of the following problem

$$
\left\{\begin{array}{l}
-x^{\prime \prime}+\rho p(t) x=0, \quad t \in(0,1), \\
c x(1)+d x^{\prime}(1)=0
\end{array}\right.
$$

In addition, if $\left(\mathrm{H}_{4}\right)$ holds, then $u(t), v(t) \in C^{1}[0,1]$ and the Wronskian

$$
\omega=\omega(t)=\left|\begin{array}{ll}
v(t) & v^{\prime}(t) \\
u(t) & u^{\prime}(t)
\end{array}\right|=\text { constant }>0
$$

where $e_{1}(t)$ and $e_{2}(t)$ are given by Lemma 1 .

Lemma 3. Suppose that $\left(\mathrm{H}_{4}\right)$ holds. Let $x(t)$ be a $C^{1}[0,1]$ positive solution of (1.1). Then there are constants $I_{1}$ and $I_{2}, 0<I_{1}<I_{2}$, such that

$$
I_{1} u(t) v(t) \leq x(t) \leq I_{2} u(t) v(t), \quad t \in[0,1]
$$

where $u(t)$ and $v(t)$ are given by Lemma 2 .

Proof. Assume that $x(t)$ is a $C^{1}[0,1]$ positive solution of (1.1). Then $x^{\prime}(0) \geq 0$ and $x^{\prime}(1) \leq 0, x(t)>0$ for $t \in(0,1)$. By integration of $(1.1)$, we have

$$
\int_{0}^{1} f(t, x(t)) d t \leq-x^{\prime}(1)+x^{\prime}(0)+\rho \max _{t \in[0,1]}|x(t)| \int_{0}^{1} p(t) d t<\infty .
$$

Let $t_{0} \in(0,1)$ and let $a_{1}$ be a constant sufficiently small satisfying $x\left(t_{0}\right)-$ $a_{1} u\left(t_{0}\right) \geq 0$, and let $y(t)=x(t)-a_{1} u(t), t \in\left[0, t_{0}\right]$. Then

$$
\left\{\begin{array}{l}
-y^{\prime \prime}(t)+\rho p(t) y(t)=f(t, x(t)) \geq 0, \quad t \in\left(0, t_{0}\right] \\
a y(0)-b y^{\prime}(0)=0, \quad y\left(t_{0}\right)=x\left(t_{0}\right)-a_{1} u\left(t_{0}\right) \geq 0
\end{array}\right.
$$


By the maximum principle, we have $y(t) \geq 0$ for $t \in\left[0, t_{0}\right]$. Therefore,

$$
x(t) \geq a_{1} u(t), \quad t \in\left[0, t_{0}\right] .
$$

On the other hand, let $a_{2}$ be a constant sufficiently large such that

$$
\begin{gathered}
a_{2} u\left(t_{0}\right)-x\left(t_{0}\right)=r_{0}, \\
r_{0} \geq\left(2 u\left(t_{0}\right) / \omega^{*}\right) \int_{0}^{t_{0}} y_{2}(0) f(s, x(s)) d s, \\
r_{0} \geq\left(2 u\left(t_{0}\right) / \omega^{*}\right) \int_{0}^{t_{0}} y_{2}(s) f(s, x(s)) d s .
\end{gathered}
$$

Here, $y_{2}(t)$ is a unique decreasing positive solution of the problem

$$
\left\{\begin{array}{l}
-y^{\prime \prime}(t)+\rho p(t) y(t)=0, \quad t \in\left(0, t_{0}\right] \\
y\left(t_{0}\right)=0, \quad y^{\prime}\left(t_{0}\right)=-1
\end{array}\right.
$$

and

$$
\omega^{*}=\left|\begin{array}{ll}
y_{2}(t) & y_{2}^{\prime}(t) \\
u(t) & u^{\prime}(t)
\end{array}\right|=\text { constant }>0 .
$$

Let $y(t)=a_{2} u(t)-x(t)$. Then

$$
\left\{\begin{array}{l}
-y^{\prime \prime}(t)+\rho p(t) y(t)=-f(t, x(t)), \quad t \in\left(0, t_{0}\right], \\
a y(0)-b y^{\prime}(0)=0, \quad y\left(t_{0}\right)=a_{2} u\left(t_{0}\right)-x\left(t_{0}\right)=r_{0}>0 .
\end{array}\right.
$$

By $\left(\mathrm{H}_{4}\right),(2.11)$ and Theorem 2.2 in [5], (2.13) has a unique solution $y(t)$ satisfying

$$
\begin{aligned}
y(t)= & \frac{u(t)}{u\left(t_{0}\right)} r_{0}-\frac{1}{\omega^{*}} \int_{0}^{t} y_{2}(t) u(s) f(s, x(s)) d s \\
& -\frac{1}{\omega^{*}} \int_{t}^{t_{0}} y_{2}(s) u(t) f(s, x(s)) d s \\
\geq & u(t)\left[\frac{r_{0}}{2 u\left(t_{0}\right)}-\frac{1}{\omega^{*}} \int_{0}^{t_{0}} y_{2}(0) f(s, x(s)) d s\right] \\
& +u(t)\left[\frac{r_{0}}{2 u\left(t_{0}\right)}-\frac{1}{\omega^{*}} \int_{0}^{t_{0}} y_{2}(s) f(s, x(s)) d s\right] \geq 0, \quad t \in\left[0, t_{0}\right] .
\end{aligned}
$$

Hence,

$$
x(t) \leq a_{2} u(t), \quad t \in\left[0, t_{0}\right] .
$$


Similarly, we can verify that there exist two numbers $b_{1}$ and $b_{2}$ satisfying

$$
b_{1} v(t) \leq x(t) \leq b_{2} v(t), \quad t \in\left[t_{0}, 1\right]
$$

For $t \in\left[0, t_{0}\right]$, from (2.12) and (2.14), we have

$$
\begin{gathered}
x(t) \geq \frac{a_{1}}{v(0)} v(0) u(t) \geq \frac{a_{1}}{v(0)} u(t) v(t), \\
x(t) \leq \frac{a_{2}}{v\left(t_{0}\right)} v\left(t_{0}\right) u(t) \leq \frac{a_{2}}{v\left(t_{0}\right)} u(t) v(t) .
\end{gathered}
$$

For $t \in\left[t_{0}, 1\right]$, from $(2.15)$, we have

$$
\begin{gathered}
x(t) \geq \frac{b_{1}}{u(1)} u(1) v(t) \geq \frac{b_{1}}{u(1)} u(t) v(t), \\
x(t) \leq \frac{b_{2}}{u\left(t_{0}\right)} u\left(t_{0}\right) v(t) \leq \frac{b_{2}}{u\left(t_{0}\right)} u(t) v(t) .
\end{gathered}
$$

Let

$$
I_{1}=\min \left\{\frac{a_{1}}{v(0)}, \quad \frac{b_{1}}{u(1)}\right\}, \quad I_{2}=\max \left\{\frac{a_{2}}{v\left(t_{0}\right)}, \frac{b_{2}}{u\left(t_{0}\right)}\right\} .
$$

Then, (2.16)-(2.19) imply that (2.10) holds. The proof of Lemma 3 is complete.

\section{$\S 3$. Main results}

A function $\alpha(t)$ is called a lower solution of (1.1) if $\alpha(t) \in C[0,1] \cap$ $C^{2}(0,1)$, and satisfies

$$
\left\{\begin{array}{l}
-\alpha^{\prime \prime}(t)+\rho p(t) \alpha(t) \leq f(t, \alpha(t)), \quad t \in(0,1) \\
a \alpha(0)-b \alpha^{\prime}(0) \leq 0, \quad c \alpha(1)+d \alpha^{\prime}(1) \leq 0
\end{array}\right.
$$

Similarly, a function $\beta(t)$ is called an upper solution of $(1.1)$ if $\beta(t) \in$ $C[0,1] \cap C^{2}(0,1)$, and satisfies

$$
\left\{\begin{array}{l}
-\beta^{\prime \prime}(t)+\rho p(t) \beta(t) \geq f(t, \beta(t)), \quad t \in(0,1), \\
a \beta(0)-b \beta^{\prime}(0) \geq 0, \quad c \beta(1)+d \beta^{\prime}(1) \geq 0 .
\end{array}\right.
$$

Now, we state the main results of this paper which are the following two theorems. 
TheOREM 3.1. Suppose that $\left(\mathrm{H}_{4}\right)$ and $\left(\mathrm{H}_{5}\right)$ hold. Then a necessary and sufficient condition for problem (1.1) to have $C^{1}[0,1]$ positive solutions is that the following inequality holds:

$$
0<\int_{0}^{1} f(t, e(t)) d t<\infty
$$

where $e(t)=u(t) v(t), u(t), v(t)$ are given by (2.5), (2.7), respectively.

THEOREM 3.2. Suppose $\left(\mathrm{H}_{5}\right)$ holds.

I) If $b=d=0$, and $\left(\mathrm{H}_{1}\right)$ holds, then a necessary and sufficient condition for problem $(1.1)$ to have $C[0,1]$ positive solutions is that the following integral conditions hold:

$$
0<\int_{0}^{1} t(1-t) f(t, 1) d t<\infty, \quad \text { also }
$$

$$
\lim _{t \rightarrow 0^{+}} t \int_{t}^{1}(1-s) f(s, 1) d s=0 \quad \text { if } \int_{0}^{1}(1-s) f(s, 1) d s=\infty
$$

and

$$
\lim _{t \rightarrow 1^{-}}(1-t) \int_{0}^{t} s f(s, 1) d s=0 \quad \text { if } \quad \int_{0}^{1} s f(s, 1) d s=\infty .
$$

II) If $b=0, d>0$, and $\left(\mathrm{H}_{2}\right)$ holds, then a necessary and sufficient condition for problem (1.1) to have $C^{1}(0,1]$ positive solutions is that the following integral conditions hold:

$$
0<\int_{0}^{1} t f(t, 1) d t<\infty, \quad \text { also }
$$

$$
\lim _{t \rightarrow 0^{+}} t \int_{t}^{1} f(s, 1) d s=0 \quad \text { if } \int_{0}^{1} f(s, 1) d s=\infty .
$$

III) If $b>0, d=0$, and $\left(\mathrm{H}_{3}\right)$ holds, then a necessary and sufficient condition for problem (1.1) to have $C^{1}[0,1)$ positive solutions is that the following integral conditions hold:

$$
\begin{gathered}
0<\int_{0}^{1}(1-t) f(t, 1) d t<\infty, \quad \text { also } \\
\lim _{t \rightarrow 1^{-}}(1-t) \int_{0}^{t} f(s, 1) d s=0 \text { if } \int_{0}^{1} f(s, 1) d s=\infty .
\end{gathered}
$$


Remark 2. When $b=d=0, p(t)=0, f(t, x)=p_{1}(t) x^{-\lambda_{1}}, \lambda_{1}>0$, we obtain the main results of paper [3]. When $b=d=0, p(t)=0, f(t, x)=$ $p_{1}(t) x^{\lambda_{1}}, 0<\lambda_{1}<1$, we get the Theorems 1 and 2 in paper [4]. When $p(t)=0, f(t, x)=p_{1}(t) x^{-\lambda_{1}}, \lambda_{1}>0$, we obtain the main results of paper [6]. When $p(t)=0, f(t, x)=p_{1}(t) x^{\lambda_{1}}, 0<\lambda_{1}<1$, we get the Theorems 1 and 2 in paper [7].

The proof of Theorem 3.1 .

1. Necessity. Suppose that $x(t)$ is a $C^{1}[0,1]$ positive solution of (1.1). Then both $x^{\prime}(0) \geq 0$ and $x^{\prime}(1) \leq 0$ exist. By Lemma 3 , there are constants $I_{1}$ and $I_{2}, 0<I_{1}<I_{2}$ such that

$$
I_{1} e(t) \leq x(t) \leq I_{2} e(t), \quad t \in[0,1]
$$

Let $c_{0}$ be a constant satisfying $c_{0} I_{2} \leq N, 1 / c_{0} \geq M$. Then (1.11), (1.12) and (3.9) lead to

$$
\begin{aligned}
f(t, x(t)) & \geq\left(1 / c_{0}\right)^{\lambda} f\left(t, \frac{c_{0} x(t)}{e(t)} e(t)\right) \\
& \geq\left(c_{0}\right)^{\mu-\lambda}\left(\frac{x(t)}{e(t)}\right)^{\mu} f(t, e(t)) \\
& \geq\left(c_{0}\right)^{\mu-\lambda} I_{1}^{\mu} f(t, e(t)), \quad t \in(0,1) .
\end{aligned}
$$

Consequently,

$$
\begin{aligned}
& 0<\int_{0}^{1} f(t, e(t)) d t \leq\left(c_{0}\right)^{\lambda-\mu} I_{1}^{-\mu} \int_{0}^{1} f(t, x(t)) d t \\
& \leq\left(c_{0}\right)^{\lambda-\mu} I_{1}^{-\mu}\left(x^{\prime}(0)-x^{\prime}(1)+I_{2} \rho v(0) u(1) \int_{0}^{1} p(t) d t\right)<\infty .
\end{aligned}
$$

Thus (3.1) holds.

2. Sufficiency. Suppose that (3.1) holds. Let

$$
h(t)=\frac{v(t)}{\omega} \int_{0}^{t} u(s) f(s, e(s)) d s+\frac{u(t)}{\omega} \int_{t}^{1} v(s) f(s, e(s)) d s, \quad t \in[0,1] .
$$

Then $h(t) \in C^{1}[0,1] \cap C^{2}(0,1)$ and (3.9) holds if $x(t)$ is replaced by $h(t)$, and

$$
I_{1}=\frac{1}{u(1) v(0) \omega} \int_{0}^{1} e(s) f(s, e(s)) d s, \quad I_{2}=\frac{1}{\omega} \int_{0}^{1} f(s, e(s)) d s
$$


Suppose that constant $c_{1}$ satisfies $c_{1} I_{1} \geq M, 1 / c_{1} \leq N$. Let $\alpha(t)=$ $k_{1} h(t), \beta(t)=k_{2} h(t), \quad t \in[0,1]$; here

$$
k_{1}=\min \left\{1, \quad\left(I_{2}^{\lambda} c_{1}^{\lambda-\mu}\right)^{1 /(1-\mu)}\right\}
$$

and

$$
k_{2}=\max \left\{1, \quad\left(I_{2}^{\mu} c_{1}^{\mu-\lambda}\right)^{1 /(1-\mu)}\right\}
$$

For $t \in(0,1)$,

$$
\begin{gathered}
f(t, \alpha(t)) \geq\left(\frac{k_{1}}{c_{1}}\right)^{\mu} f\left(t, \frac{c_{1} h(t)}{e(t)} e(t)\right) \geq k_{1}^{\mu} c_{1}^{\lambda-\mu} I_{2}^{\lambda} f(t, e(t)), \\
f(t, \beta(t)) \leq\left(\frac{1}{c_{1}}\right)^{\lambda} f\left(t, \frac{k_{2} c_{1} h(t)}{e(t)} e(t)\right) \leq k_{2}^{\mu} c_{1}^{\mu-\lambda} I_{2}^{\mu} f(t, e(t)), \\
-\alpha^{\prime \prime}(t)+\rho p(t) \alpha(t)=k_{1} f(t, e(t)) \leq k_{1}^{\mu} c_{1}^{\lambda-\mu} I_{2}^{\lambda} f(t, e(t)) \leq f(t, \alpha(t)), \\
-\beta^{\prime \prime}(t)+\rho p(t) \beta(t)=k_{2} f(t, e(t)) \geq k_{2}^{\mu} c_{1}^{\mu-\lambda} I_{2}^{\mu} f(t, e(t)) \geq f(t, \beta(t)) .
\end{gathered}
$$

So, $\alpha(t), \beta(t) \in C^{1}[0,1] \cap C^{2}(0,1)$ are, respectively, lower and upper solutions of (1.1) satisfying $0<\alpha(t) \leq \beta(t)$ for $t \in(0,1)$, and $a \alpha(0)-b \alpha^{\prime}(0)=$ $0, c \alpha(1)+d \alpha^{\prime}(1)=0, a \beta(0)-b \beta^{\prime}(0)=0, c \beta(1)+d \beta^{\prime}(1)=0$. Additionally, when $t \in(0,1)$ and $\alpha(t) \leq x \leq \beta(t)$, we have

$$
\begin{aligned}
0 & \leq f(t, x) \leq\left(\frac{k_{1}}{c_{1}}\right)^{\lambda} f\left(t, \frac{c_{1} x}{k_{1} e(t)} e(t)\right) \\
& \leq\left(\frac{k_{1}}{c_{1}}\right)^{\lambda}\left(\frac{c_{1} x}{k_{1} e(t)}\right)^{\mu} f(t, e(t)) \\
& \leq\left(\frac{k_{1}}{c_{1}}\right)^{\lambda-\mu}\left(k_{2} I_{2}\right)^{\mu} f(t, e(t))=F(t) .
\end{aligned}
$$

From (3.1), we have $\int_{0}^{1} F(t) d t<\infty$. In the following, we shall show that problem (1.1) admits a positive solution $x(t) \in C^{1}[0,1] \cap C^{2}(0,1)$ such that $\alpha(t) \leq x(t) \leq \beta(t)$ for $t \in[0,1]$

First of all, we define an auxiliary function

$$
g(t, x)=\left\{\begin{array}{lll}
f(t, \alpha(t)), & \text { if } & x<\alpha(t), \\
f(t, x), & \text { if } & \alpha(t) \leq x \leq \beta(t), \\
f(t, \beta(t)), & \text { if } & x>\beta(t) .
\end{array}\right.
$$


Consider the singular problem

$$
\left\{\begin{array}{l}
-x^{\prime \prime}+\rho p(t) x=g(t, x), \quad t \in(0,1), \\
a x(0)-b x^{\prime}(0)=0, \quad c x(1)+d x^{\prime}(1)=0,
\end{array}\right.
$$

and the corresponding integral equation

$$
x(t)=A x(t)=\int_{0}^{1} G(t, s) g(s, x(s)) d s,
$$

where

$$
G(t, s)= \begin{cases}\frac{v(t) u(s)}{\omega}, & s<t \\ \frac{v(s) u(t)}{\omega}, & t \leq s\end{cases}
$$

$\omega$ is given by (2.9). Obviously, if $x \in C[0,1] \cap C^{1}[0,1]$ is a solution of (3.13), then $x$ is a $C^{1}[0,1]$ solution of $(3.12)$.

By virtue of (3.1), (3.10) and (3.11), it is easy to verify that $A: X \rightarrow$ $X=C[0,1]$ is completely continuous and $A(X)$ is a bounded set. Using the Schauder fixed point theorem, we assert that $A$ has at least one fixed point $x^{*} \in X \cap C^{1}[0,1]$.

We claim that

$$
\alpha(t) \leq x^{*}(t) \leq \beta(t), \quad t \in[0,1]
$$

and hence $x^{*}(t) \in C^{1}[0,1]$ is a positive solution of (1.1). Indeed, suppose by contradiction that there is $t^{*} \in[0,1]$ such that $x^{*}\left(t^{*}\right)>\beta\left(t^{*}\right)$. Then the relationships between $x(t)$ and $\beta(t)$ must be one of the following four cases:

Case 1: $x^{*}(t)>\beta(t), t \in[0,1]$;

Case 2: there exists $0<s \leq 1$ such that $x^{*}(s)=\beta(s), \quad x^{*}(t)>$ $\beta(t), t \in[0, s)$, and $t^{*} \in[0, s)$;

Case 3: there exists $0 \leq r<1$ such that $x^{*}(r)=\beta(r), \quad x^{*}(t)>$ $\beta(t), t \in(r, 1]$, and $t^{*} \in(r, 1]$;

Case 4: there exist $0 \leq r<s \leq 1$ such that $x^{*}(r)=\beta(r), \quad x^{*}(s)=$ $\beta(s), \quad x^{*}(t)>\beta(t), t \in(r, s)$, and $t^{*} \in(r, s)$.

For the Case 1: for $t \in[0,1]$, we have that $g\left(t, x^{*}(t)\right)=f(t, \beta(t))$ and therefore

$$
-x^{*^{\prime \prime}}(t)+\rho p(t) x^{*}(t)=f(t, \beta(t)), \quad t \in(0,1) .
$$


On the other hand, as $\beta$ is an upper solution of (1.1), we also have

$$
-\beta^{\prime \prime}(t)+\rho p(t) \beta(t) \geq f(t, \beta(t)), \quad t \in(0,1) .
$$

Then, setting

$$
z(t)=\beta(t)-x^{*}(t), \quad t \in[0,1]
$$

we obtain $-z^{\prime \prime}(t)+\rho p(t) z(t) \geq 0, t \in[0,1]$, and $a z(0)-b z^{\prime}(0)=0, \quad c z(1)+$ $d z^{\prime}(1)=0$. By the maximum principle, we can conclude that $z(t) \geq 0, t \in$ $[0,1]$, that is $\beta(t) \geq x^{*}(t), t \in[0,1]$, a contradiction to the assumption $\beta\left(t^{*}\right)<x^{*}\left(t^{*}\right)$. The proof for the cases 2,3 and 4 is analogous to that of the case 1. Similarly, we can show that $\alpha(t) \leq x^{*}(t), \quad t \in[0,1]$. Therefore, (3.15) holds, and $x^{*}(t)$ is a $C^{1}[0,1]$ positive solution of $(1.1)$. The proof of Theorem 3.1 is complete.

The proof of Theorem 3.2. The proof for the case I): $b=d=0$.

1. Necessity. Let $x(t) \in C[0,1]$ be a positive solution of (1.1). Then $x(0)=x(1)=0$ and there is a $t_{0} \in(0,1)$ such that $x^{\prime}\left(t_{0}\right)=0$. Let $c_{0}>0$ be a constant such that $c_{0} x(t) \leq N$ for $t \in[0,1]$ and $1 / c_{0} \geq M$. From (1.11) and (1.12), we have

$$
f(t, x(t)) \geq\left(1 / c_{0}\right)^{\lambda} f\left(t, c_{0} x(t)\right) \geq c_{0}^{\mu-\lambda} x^{\mu}(t) f(t, 1) \quad \text { for } \quad t \in(0,1) .
$$

According to (1.1), we have

$$
c_{0}^{\mu-\lambda} f(t, 1) \leq-x^{-\mu}(t) x^{\prime \prime}(t)+\rho p(t) x^{1-\mu}(t), \quad t \in(0,1) .
$$

For $t \in\left(0, t_{0}\right)$, by integration of $(3.16)$, we obtain

$$
\begin{aligned}
c_{0}^{\mu-\lambda} \int_{t}^{t_{0}} f(s, 1) d s \leq & -\left.x^{\prime}(s) x^{-\mu}(s)\right|_{t} ^{t_{0}}+\int_{t}^{t_{0}}\left(-\mu x^{-\mu-1}(s)\right)\left(x^{\prime}(s)\right)^{2} d s \\
& +\rho \int_{t}^{t_{0}} p(s) x^{1-\mu}(s) d s \\
\leq & x^{-\mu}(t) x^{\prime}(t)+\rho \int_{t}^{t_{0}} p(s) x^{1-\mu}(s) d s, \quad t \in\left(0, t_{0}\right) .
\end{aligned}
$$

Integrating (3.17), we have

$$
\begin{aligned}
& c_{0}^{\mu-\lambda} \int_{0}^{t_{0}} \int_{t}^{t_{0}} f(s, 1) d s d t \leq \frac{x^{1-\mu}\left(t_{0}\right)}{1-\mu}+\rho K \int_{0}^{t_{0}} \int_{t}^{t_{0}} p(s) d s d t \\
& =\frac{x^{1-\mu}\left(t_{0}\right)}{1-\mu}+\rho K \int_{0}^{t_{0}} s p(s) d s<\infty
\end{aligned}
$$


where $K=\max _{t \in[0,1]} x^{1-\mu}(t)$, so,

$$
0<\int_{0}^{t_{0}} s f(s, 1) d s<\infty .
$$

For $t \in\left(t_{0}, 1\right)$, by integration of $(3.16)$, we obtain

$$
c_{0}^{\mu-\lambda} \int_{t_{0}}^{t} f(s, 1) d s \leq-x^{-\mu}(t) x^{\prime}(t)+\rho K \int_{t_{0}}^{t} p(s) d s, \quad t \in\left(t_{0}, 1\right) .
$$

By integration (3.19), we have

$$
c_{0}^{\mu-\lambda} \int_{t_{0}}^{1} \int_{t_{0}}^{t} f(s, 1) d s d t \leq \frac{x^{1-\mu}\left(t_{0}\right)}{1-\mu}+\rho K \int_{t_{0}}^{1}(1-s) p(s) d s<\infty,
$$

i.e.,

$$
0<\int_{t_{0}}^{1}(1-s) f(s, 1) d s<\infty .
$$

Then, (3.18) and (3.20) imply that (3.2) holds.

For $t \in\left(0, t_{0}\right)$, by integration of $(3.17)$, we have

$$
\begin{aligned}
& c_{0}^{\mu-\lambda} \int_{0}^{t} \int_{s}^{t_{0}} f(\tau, 1) d \tau d s \leq \frac{x^{1-\mu}(t)}{1-\mu}+\rho K \int_{0}^{t} \int_{s}^{t_{0}} p(\tau) d \tau d s \\
& =\frac{x^{1-\mu}(t)}{1-\mu}+\rho K \int_{0}^{t} d s\left(\int_{s}^{t}+\int_{t}^{t_{0}}\right) p(\tau) d \tau
\end{aligned}
$$

therefore,

$$
c_{0}^{\mu-\lambda} t \int_{t}^{t_{0}} f(\tau, 1) d \tau \leq \frac{x^{1-\mu}(t)}{1-\mu}+\rho K\left(\int_{0}^{t} s p(s) d s+t \int_{t}^{t_{0}} p(\tau) d \tau\right)
$$

Letting $t \rightarrow 0$ in (3.21) and noting condition $\left(\mathrm{H}_{1}\right)$ and $x(0)=0$, we have

$$
\lim _{t \rightarrow 0^{+}} t \int_{t}^{t_{0}} f(s, 1) d s=0 .
$$

These imply that (3.3) holds.

For $t \in\left(t_{0}, 1\right)$, by integration of $(3.19)$, we have

$$
c_{0}^{\mu-\lambda} \int_{t}^{1} \int_{t_{0}}^{s} f(\tau, 1) d \tau d s \leq \frac{x^{1-\mu}(t)}{1-\mu}+\rho K \int_{t}^{1} d s\left(\int_{t_{0}}^{t}+\int_{t}^{s}\right) p(\tau) d \tau .
$$


Therefore,

$$
\begin{aligned}
& \quad(1-t) \int_{t_{0}}^{t} f(\tau, 1) d \tau \\
& \leq c_{0}^{\lambda-\mu}\left(\frac{x^{1-\mu}(t)}{1-\mu}+\rho K\left((1-t) \int_{t_{0}}^{t} p(\tau) d \tau+\int_{t}^{1}(1-\tau) p(\tau) d \tau\right)\right) .
\end{aligned}
$$

Letting $t \rightarrow 1$ in (3.23) and noting condition $\left(\mathrm{H}_{1}\right)$ and $x(1)=0$, we obtain

$$
\lim _{t \rightarrow 1^{-}}(1-t) \int_{t_{0}}^{t} f(s, 1) d s=0 .
$$

These imply that (3.4) holds.

2. Sufficiency. Suppose that (3.2)-(3.4) hold. By Theorem 2.2 in [5], we know

$$
\omega_{0}=e_{2}(0)=e_{1}(1)=\left|\begin{array}{ll}
e_{2}(t) & e_{2}^{\prime}(t) \\
e_{1}(t) & e_{1}^{\prime}(t)
\end{array}\right|=\text { constant }>0
$$

Here, $e_{1}(t), e_{2}(t)$ are given by Lemma 1 . Choose a constant $m \geq 2$ such that $m(\mu-\lambda)>1$, and let

$$
q(t)=\frac{1}{\omega_{0}}\left(e_{2}(t) \int_{0}^{t} e_{1}(s) f(s, 1) d s+e_{1}(t) \int_{t}^{1} e_{2}(s) f(s, 1) d s\right)
$$

$$
Q(t)=(q(t))^{1 /(m(\mu-\lambda))}
$$

Then $q(t), Q(t) \in C[0,1] \cap C^{2}(0,1)$ satisfying $q(t)>0, Q(t)>0, t \in(0,1)$, and

$$
-q^{\prime \prime}(t)+\rho p(t) q(t)=f(t, 1), \quad-Q^{\prime \prime}(t)+\rho p(t) Q(t) \geq 0, \text { for } t \in(0,1)
$$

and from $(3.2)-(3.4)$, we have $q(i)=Q(i)=0$, for $i=0,1$. By the proof of Lemma 1, we obtain

$$
0<q(t) \leq \frac{1}{\omega_{0}} \int_{0}^{1} s(1-s) w_{1}(1) w_{2}(0) f(s, 1) d s<\infty, \quad t \in(0,1)
$$


and such that

$$
\begin{aligned}
& e_{2}(t) \int_{0}^{t} e_{1}(s) Q^{-(\mu-\lambda)}(s) f(s, 1) d s \\
& \leq e_{2}(t) \int_{0}^{t} e_{1}(s)\left(\frac{e_{2}(s)}{\omega_{0}} \int_{0}^{s} e_{1}(\tau) f(\tau, 1) d \tau\right)^{-1 / m} f(s, 1) d s \\
&(3.29) \quad \leq\left(e_{2}(t)\right)^{1-1 / m} \omega_{0}^{1 / m} \int_{0}^{t} e_{1}(s)\left(\int_{0}^{s} e_{1}(\tau) f(\tau, 1) d \tau\right)^{-1 / m} f(s, 1) d s \\
& \quad=\omega_{0}^{1 / m}(1-1 / m)^{-1}\left(e_{2}(t)\right)^{1-1 / m}\left(\int_{0}^{t} e_{1}(s) f(s, 1) d s\right)^{1-1 / m} \\
& \leq \omega_{0}^{1 / m}(1-1 / m)^{-1}\left(\int_{0}^{1} e_{1}(s) e_{2}(s) f(s, 1) d s\right)^{1-1 / m}<\infty .
\end{aligned}
$$

Similarly, we have

$$
\begin{aligned}
& e_{1}(t) \int_{t}^{1} e_{2}(s) Q^{-(\mu-\lambda)}(s) f(s, 1) d s \\
& \leq \omega_{0}^{1 / m}(1-1 / m)^{-1}\left(\int_{0}^{1} e_{1}(s) e_{2}(s) f(s, 1) d s\right)^{1-1 / m}<\infty .
\end{aligned}
$$

Let

$$
\begin{aligned}
h_{1}(t)= & \frac{e_{2}(t)}{\omega_{0}} \int_{0}^{t} e_{1}(s)\left(\frac{e_{1}(s) e_{2}(s)}{e_{1}(1) e_{2}(0)}\right)^{\mu} f(s, 1) d s \\
& +\frac{e_{1}(t)}{\omega_{0}} \int_{t}^{1} e_{2}(s)\left(\frac{e_{1}(s) e_{2}(s)}{e_{1}(1) e_{2}(0)}\right)^{\mu} f(s, 1) d s \\
h_{2}(t)= & \frac{e_{2}(t)}{\omega_{0}} \int_{0}^{t} e_{1}(s) Q^{-\mu}(s) f(s, Q(s)) d s \\
& +\frac{e_{1}(t)}{\omega_{0}} \int_{t}^{1} e_{2}(s) Q^{-\mu}(s) f(s, Q(s)) d s+Q(t) .
\end{aligned}
$$

Let $c_{1}>0$ such that $\left(1 / c_{1}\right) Q(t) \leq N<1, \quad c_{1} \geq M>1$. From (1.11) and (1.12), we have

$$
\begin{aligned}
& Q^{-\mu}(t) f(t, Q(t)) \leq Q^{-\mu}(t)\left(Q(t) / c_{1}\right)^{\lambda} f\left(t, c_{1}\right) \\
& \leq Q^{-\mu}(t)\left(Q(t) / c_{1}\right)^{\lambda} c_{1}^{\mu} f(t, 1)=c_{1}^{\mu-\lambda} Q^{\lambda-\mu}(t) f(t, 1) .
\end{aligned}
$$


Thus, (3.28)-(3.31) imply that

$$
0 \leq h_{1}(t)<\infty, \quad 0 \leq h_{2}(t)<\infty, \quad \text { for } \quad t \in[0,1]
$$

One can check that $h_{i} \in C[0,1] \cap C^{2}(0,1), h_{i}(0)=h_{i}(1)=0, \quad i=1,2$, and

$$
\begin{gathered}
L_{1} \frac{e_{1}(t) e_{2}(t)}{e_{1}(1) e_{2}(0)} \leq h_{1}(t) \leq L_{1}, \quad Q(t) \leq h_{2}(t) \leq L_{2}, \quad t \in[0,1], \\
-h_{1}^{\prime \prime}(t)+\rho p(t) h_{1}(t)=\left(\frac{e_{1}(t) e_{2}(t)}{e_{1}(1) e_{2}(0)}\right)^{\mu} f(t, 1), \quad t \in(0,1), \\
-h_{2}^{\prime \prime}(t)+\rho p(t) h_{2}(t) \geq Q^{-\mu}(t) f(t, Q(t)), \quad t \in(0,1) .
\end{gathered}
$$

Here,

$$
\begin{gathered}
L_{1}=\omega_{0} \int_{0}^{1}\left(\frac{e_{1}(s) e_{2}(s)}{e_{1}(1) e_{2}(0)}\right)^{1+\mu} f(s, 1) d s \\
L_{2}=\frac{1}{\omega_{0}} \int_{0}^{1} e_{1}(s) e_{2}(s) Q^{-\mu}(s) f(s, Q(s)) d s+Q_{0}, \quad Q_{0}=\max _{t \in[0,1]} Q(t) .
\end{gathered}
$$

Let $\alpha(t)=k_{1} h_{1}(t), \beta(t)=k_{2} h_{2}(t), \quad t \in[0,1]$; here $k_{1}, k_{2}$ are constants satisfying $0<k_{1} \leq 1 \leq k_{2}$ and will be determined later. Suppose $c_{2}, c_{3}$ are constants such that $c_{2} L_{1} \leq N, 1 / c_{2} \geq M, c_{3} \geq M, 1 / c_{3} \leq N$. From (1.11), (1.12), we have

$$
\begin{aligned}
f(t, \alpha(t)) & \geq\left(1 / c_{2}\right)^{\lambda} f\left(t, c_{2} \alpha(t)\right) \geq\left(c_{2}\right)^{\mu-\lambda} \alpha^{\mu}(t) f(t, 1) \\
& \geq\left(c_{2}\right)^{\mu-\lambda}\left(k_{1} L_{1}\right)^{\mu}\left(\frac{e_{1}(t) e_{2}(t)}{e_{1}(1) e_{2}(0)}\right)^{\mu} f(t, 1), \quad t \in(0,1), \\
f(t, \beta(t)) & \leq\left(c_{3}\right)^{\mu-\lambda}\left(\frac{\beta(t)}{Q(t)}\right)^{\mu} f(t, Q(t)) \\
& \leq\left(c_{3}\right)^{\mu-\lambda}\left(k_{2} L_{2}\right)^{\mu} Q^{-\mu}(t) f(t, Q(t)), \quad t \in(0,1) .
\end{aligned}
$$

By virtue of (1.11), (1.12), we can find a $k_{0}$ such that $f(t, Q(t)) \geq$ $k_{0} Q^{\mu}(t) f(t, 1)$, and hence, from the definitions of $h_{1}(t), h_{2}(t)$, we have $h_{1}(t) \leq k_{0}^{-1} h_{2}(t)$ for $t \in[0,1]$. Now we choose

$$
k_{1}=\min \left\{1, \quad\left(L_{1}^{\mu} c_{2}^{\mu-\lambda}\right)^{1 /(1-\mu)}\right\}
$$


and

$$
k_{2}=\max \left\{1, \quad k_{0}^{-1}, \quad\left(L_{2}^{\mu} c_{3}^{\mu-\lambda}\right)^{1 /(1-\mu)}\right\} .
$$

Then $\alpha(t), \beta(t) \in C[0,1] \cap C^{2}(0,1), \quad 0<\alpha(t) \leq \beta(t)$ for $t \in(0,1), \alpha(i)=$ $\beta(i)=0, \quad i=0,1$. From (3.32)-(3.35), we obtain that for such choice of $k_{1}$ and $k_{2}, \alpha(t)$ and $\beta(t)$ are lower and upper solutions of (1.1), respectively.

In the following, we shall prove problem (1.1) has at least one $C[0,1]$ positive solution $x(t)$ such that

$$
\alpha(t) \leq x(t) \leq \beta(t), \quad t \in[0,1] .
$$

First of all, we define an auxiliary function $g(t, x)$ given by (3.11). Let $\left\{a_{n}\right\}, \quad\left\{b_{n}\right\}$ be sequences satisfying $0<\cdots<a_{n+1}<a_{n}<\cdots<a_{1}<$ $1 / 2<b_{1}<\cdots<b_{n}<b_{n+1}<\cdots<1, a_{n} \rightarrow 0$ and $b_{n} \rightarrow 1$ as $n \rightarrow \infty$, and let $\left\{r_{1}^{(n)}\right\}, \quad\left\{r_{2}^{(n)}\right\}$ be sequences satisfying

$$
\alpha\left(a_{n}\right) \leq r_{1}^{(n)} \leq \beta\left(a_{n}\right), \quad \alpha\left(b_{n}\right) \leq r_{2}^{(n)} \leq \beta\left(b_{n}\right), \quad n=1,2, \ldots
$$

For each $n$, consider the nonsingular problem

$$
\left\{\begin{array}{l}
-x^{\prime \prime}+\rho p(t) x=g(t, x), \quad t \in\left[a_{n}, b_{n}\right], \\
x\left(a_{n}\right)=r_{1}^{(n)}, \quad x\left(b_{n}\right)=r_{2}^{(n)}
\end{array}\right.
$$

and the corresponding integral equation

$$
x(t)=A_{n} x(t)=\frac{x_{2 n}(t)}{x_{2 n}\left(a_{n}\right)} r_{1}^{(n)}+\frac{x_{1 n}(t)}{x_{1 n}\left(b_{n}\right)} r_{2}^{(n)}+\int_{a_{n}}^{b_{n}} G_{n}(t, s) g(s, x(s)) d s,
$$

where

$$
\begin{gathered}
G_{n}(t, s)= \begin{cases}\frac{x_{2 n}(t) x_{1 n}(s)}{\omega_{n}}, & s<t, \\
\frac{x_{2 n}(s) x_{1 n}(t)}{\omega_{n}}, & t \leq s,\end{cases} \\
\omega_{n}=\left|\begin{array}{ll}
x_{2 n}(t) & x_{2 n}^{\prime}(t) \\
x_{1 n}(t) & x_{1 n}^{\prime}(t)
\end{array}\right|=x_{2 n}\left(a_{n}\right)=x_{1 n}\left(b_{n}\right)=\text { constant }>0,
\end{gathered}
$$

and $x_{1 n}(t) \in C^{2}\left[a_{n}, b_{n}\right]$ is a unique increasing positive solution of the problem

$$
\left\{\begin{array}{l}
-x^{\prime \prime}(t)+\rho p(t) x(t)=0, \quad t \in\left[a_{n}, b_{n}\right] \\
x\left(a_{n}\right)=0, \quad x^{\prime}\left(a_{n}\right)=1
\end{array}\right.
$$


and $x_{2 n}(t) \in C^{2}\left[a_{n}, b_{n}\right]$ is a unique decreasing positive solution of the problem

$$
\left\{\begin{array}{l}
-x^{\prime \prime}(t)+\rho p(t) x(t)=0, \quad t \in\left[a_{n}, b_{n}\right] \\
x\left(b_{n}\right)=0, \quad x^{\prime}\left(b_{n}\right)=-1
\end{array}\right.
$$

It is easy to verify that $A_{n}: X_{n} \rightarrow X_{n}=C\left[a_{n}, b_{n}\right]$ is completely continuous and $A_{n}\left(X_{n}\right)$ is a bounded set. Moreover, if $x \in C^{2}\left[a_{n}, b_{n}\right]$ is a solution of (3.38), then $x$ is a solution of (3.37). Using the Schauder fixed point theorem, we assert that $A_{n}$ has at least one fixed point $x_{n} \in C^{2}\left[a_{n}, b_{n}\right]$.

Similarly to the proof of Theorem 3.1, we can prove that $\alpha(t) \leq x_{n}(t) \leq$ $\beta(t), \quad t \in\left[a_{n}, b_{n}\right]$ and hence $x_{n}(t) \in C^{2}\left[a_{n}, b_{n}\right]$ satisfies

$$
-x_{n}^{\prime \prime}(t)+\rho p(t) x_{n}(t)=f\left(t, x_{n}(t)\right), \quad t \in\left[a_{n}, b_{n}\right] .
$$

Since $\left[a_{1}, b_{1}\right] \subset\left[a_{n}, b_{n}\right], n=1,2, \ldots$, there is, for each $n, t_{n} \in\left[a_{1}, b_{1}\right]$ such that $\left|x_{n}^{\prime}\left(t_{n}\right)\right|=\left|\left(x_{n}\left(b_{1}\right)-x_{n}\left(a_{1}\right)\right) /\left(b_{1}-a_{1}\right)\right| \leq\left(2 /\left(b_{1}-a_{1}\right)\right)\left(\beta\left(b_{1}\right)+\right.$ $\left.\beta\left(a_{1}\right)\right)$. This allows us to assume (substituting by subsequences if necessary) $t_{n} \rightarrow t_{0} \in\left[a_{n}, b_{n}\right], x_{n}\left(t_{n}\right) \rightarrow x_{0} \in\left[\alpha\left(t_{0}\right), \beta\left(t_{0}\right)\right], x_{n}^{\prime}\left(t_{n}\right) \rightarrow x_{0}^{\prime} \in R$, as $n \rightarrow$ $\infty$.

From [8, Theorem 3.2, p.14], there is a solution $x(t)$ of the equation

$$
-x^{\prime \prime}+\rho p(t) x=f(t, x)
$$

with the maximum existence interval $\left(\omega^{-}, \omega^{+}\right)$such that $x\left(t_{0}\right)=x_{0}$, $x^{\prime}\left(t_{0}\right)=x_{0}^{\prime}$ and there is a subsequence of $\left\{x_{n}(t)\right\}$ - we denote it again by $\left\{x_{n}(t)\right\}$ - such that $\left\{x_{n}(t)\right\}$ converges uniformly to $x(t)$ on any compact subintervals of $\left(\omega^{-}, \omega^{+}\right)$. Because $\left[a_{n}, b_{n}\right] \subset\left[a_{n+1}, b_{n+1}\right], \bigcup_{n=1}^{\infty}\left[a_{n}, b_{n}\right]=$ $(0,1)$, and $\alpha(t) \leq x_{n}(t) \leq \beta(t), \quad t \in\left[a_{n}, b_{n}\right]$, one can easily see that $\alpha(t) \leq x(t) \leq \beta(t)$ for $t \in\left(\omega^{-}, \omega^{+}\right)$. This leads additionally to the fact that $\left(\omega^{-}, \omega^{+}\right)=(0,1)$, from the Extension Theorem. Also, $x(t)$ satisfies $x(0)=0, \quad x(1)=0$, because $\alpha(t)$ and $\beta(t)$ do. Thus $x(t)$ is a $C[0,1]$ positive solution of problem (1.1).

This completes the proof of Theorem 3.2 for the case I): $b=d=0$.

The proof for the case II): $b=0, d>0$

1. Necessity. Let $x(t) \in C[0,1] \cap C^{1}(0,1]$ be a positive solution of (1.1). Then $x(0)=0$. By the proof of Lemma 3, we see that $x(t)$ satisfies (2.15). And (2.15) implies $x(1)>0, x^{\prime}(1) \leq 0$. Then there is a $t_{0} \in(0,1]$ 
such that $x^{\prime}\left(t_{0}\right)=0$. Hence, there are two cases, 1): $0<t_{0}<1$ and 2): $t_{0}=1$.

For the case 1): $0<t_{0}<1$, let $c_{0}>0$ be a constant such that $c_{0} x(t) \leq$ $N$ for $t \in[0,1]$ and $1 / c_{0} \geq M$. Then (3.16)-(3.22) hold. By integration of (3.16), we obtain

$$
c_{0}^{\mu-\lambda} \int_{t_{0}}^{1} f(s, 1) d s \leq-x^{-\mu}(1) x^{\prime}(1)+\rho K \int_{t_{0}}^{1} p(s) d s<\infty
$$

where $K=\max _{t \in[0,1]} x^{1-\mu}(t)$. Then, (3.18) and (3.43) imply that (3.5) holds, and (3.22) and (3.43) imply that (3.6) holds.

For the case 2): $t_{0}=1$ is similar to that of the case 1$): 0<t_{0}<1$.

2. Sufficiency. Suppose that (3.5) and (3.6) hold. By Theorem 2.2 in [5], we know

$$
\omega=\left|\begin{array}{ll}
v(t) & v^{\prime}(t) \\
e_{1}(t) & e_{1}^{\prime}(t)
\end{array}\right|=\text { constant }>0 .
$$

Here, $e_{1}(t), v(t)$ are given by Lemmas 1,2 respectively. Choose a constant $m \geq 2$ such that $m(\mu-\lambda)>1$, and let

$$
\begin{gathered}
q(t)=\frac{1}{\omega}\left(v(t) \int_{0}^{t} e_{1}(s) f(s, 1) d s+e_{1}(t) \int_{t}^{1} v(s) f(s, 1) d s\right) \\
Q(t)=(q(t))^{1 /(m(\mu-\lambda))} .
\end{gathered}
$$

We can check that $q, Q \in C[0,1] \cap C^{1}(0,1] \cap C^{2}(0,1), q(0)=Q(0)=0$, $c q(1)+d q^{\prime}(1)=0, c Q(1)+d Q^{\prime}(1) \geq 0$. Let

$$
\begin{aligned}
h_{1}(t)= & \frac{v(t)}{\omega} \int_{0}^{t} e_{1}(s)\left(\frac{e_{1}(s) v(s)}{e_{1}(1) v(0)}\right)^{\mu} f(s, 1) d s \\
& +\frac{e_{1}(t)}{\omega} \int_{t}^{1} v(s)\left(\frac{e_{1}(s) v(s)}{e_{1}(1) v(0)}\right)^{\mu} f(s, 1) d s \\
h_{2}(t)= & \frac{v(t)}{\omega} \int_{0}^{t} e_{1}(s) Q^{-\mu}(s) f(s, Q(s)) d s \\
& +\frac{e_{1}(t)}{\omega} \int_{t}^{1} v(s) Q^{-\mu}(s) f(s, Q(s)) d s+Q(t) .
\end{aligned}
$$


Then $h_{i} \in C[0,1] \cap C^{1}(0,1] \cap C^{2}(0,1), h_{i}(0)=0, i=1,2, c h_{1}(1)+d h_{1}^{\prime}(1)=$ $0, c h_{2}(1)+d h_{2}^{\prime}(1)=c Q(1)+d Q^{\prime}(1) \geq 0$. Let

$$
\begin{gathered}
L_{1}=\frac{e_{1}(1) v(0)}{\omega} \int_{0}^{1}\left(\frac{e_{1}(s) v(s)}{e_{1}(1) v(0)}\right)^{1+\mu} f(s, 1) d s \\
L_{2}=\frac{1}{\omega} \int_{0}^{1} e_{1}(s) v(s) Q^{-\mu}(s) f(s, Q(s)) d s+Q_{0}, \quad Q_{0}=\max _{t \in[0,1]} Q(t) .
\end{gathered}
$$

By virtue of (1.11), (1.12), we can find a $k_{0}$ such that $f(t, Q(t)) \geq$ $k_{0} Q^{\mu}(t) f(t, 1)$, and hence, from the definitions of $h_{1}(t), h_{2}(t)$, we have $h_{1}(t) \leq k_{0}^{-1} h_{2}(t)$ for $t \in[0,1]$. Suppose $c_{2}, c_{3}$ are constants such that $c_{2} L_{1} \leq N, 1 / c_{2} \geq M, c_{3} \geq M, 1 / c_{3} \leq N$. Now we choose

$$
k_{1}=\min \left\{1, \quad\left(L_{1}^{\mu} c_{2}^{\mu-\lambda}\right)^{1 /(1-\mu)}\right\}
$$

and

$$
k_{2}=\max \left\{1, k_{0}^{-1}, \quad\left(L_{2}^{\mu} c_{3}^{\mu-\lambda}\right)^{1 /(1-\mu)}\right\} .
$$

Let $\alpha(t)=k_{1} h_{1}(t), \beta(t)=k_{2} h_{2}(t), \quad t \in[0,1]$. A similar argument to that we have checked in the sufficiency proof of case I): $b=d=0$ in Theorem 3.2 yields $\alpha(t), \beta(t) \in C^{1}(0,1] \cap C^{2}(0,1), \quad 0<\alpha(t) \leq \beta(t)$ for $t \in(0,1]$, $\alpha(0)=\beta(0)=0, \quad c \alpha(1)+d \alpha^{\prime}(1)=0, \quad c \beta(1)+d \beta^{\prime}(1) \geq 0, \quad \alpha(t)$ and $\beta(t)$ are lower and upper solutions of (1.1), respectively.

In the following, we shall prove problem (1.1) has at least one $C[0,1] \cap$ $C^{1}(0,1]$ positive solution $x(t)$ such that

$$
\alpha(t) \leq x(t) \leq \beta(t), \quad t \in[0,1]
$$

First of all, we define an auxiliary function $g(t, x)$ given by (3.11). Let $\left\{a_{n}\right\}$ be a sequence satisfying $0<\cdots<a_{n+1}<a_{n}<\cdots<a_{1}<1 / 2, a_{n} \rightarrow 0$ as $n \rightarrow \infty$, and let $\left\{r_{1}^{(n)}\right\}$ be a sequence satisfying

$$
\alpha\left(a_{n}\right) \leq r_{1}^{(n)} \leq \beta\left(a_{n}\right), \quad n=1,2, \ldots
$$

For each $n$, consider the singular problem

$$
\left\{\begin{array}{l}
-x^{\prime \prime}+\rho p(t) x=g(t, x), \quad t \in\left[a_{n}, 1\right), \\
x\left(a_{n}\right)=r_{1}^{(n)}, \quad c x(1)+d x^{\prime}(1)=0 .
\end{array}\right.
$$


Then there exist constants $K_{n}, J$ such that $0<K_{n} \leq \alpha(t) \leq \beta(t) \leq J$ for $t \in\left[a_{n}, 1\right]$. Take constants $c_{n}$ such that $c_{n} \geq M, K_{n} / c_{n} \leq N$. Then when $t \in\left[a_{n}, 1\right], \alpha(t) \leq x \leq \beta(t)$, we have

$$
0 \leq f(t, x)=f\left(t, \frac{c_{n} x}{K_{n}} \frac{K_{n}}{c_{n}}\right) \leq\left(\frac{c_{n} J}{K_{n}}\right)^{\mu}\left(\frac{K_{n}}{c_{n}}\right)^{\lambda} f(t, 1)=F(t)
$$

Therefore,

$$
0 \leq \int_{a_{n}}^{1} F(s) d s \leq \frac{1}{a_{n}} \int_{a_{n}}^{1} s F(s) d s<\infty .
$$

By virtue of the proof of the sufficiency of Theorem 3.1, noting (3.49) and (3.50), we can obtain the following conclusion: For each $n$, the singular problem (3.48) has at least a positive solution $x_{n} \in C^{1}\left[a_{n}, 1\right]$ such that $\alpha(t) \leq x_{n}(t) \leq \beta(t), \quad t \in\left[a_{n}, 1\right]$. Hence, we have $\left|x_{n}(1)\right| \leq \beta(1)$, $\left|x_{n}^{\prime}(1)\right| \leq\left|(c / d) x_{n}(1)\right| \leq(c / d) \beta(1), n=1,2, \ldots$, This allows us to assume (substituting by subsequences if necessary) $x_{n}(1) \rightarrow x_{0} \in[\alpha(1), \beta(1)]$, $x_{n}^{\prime}(1) \rightarrow-(c / d) x_{0}$, as $n \rightarrow \infty$.

From [8, Theorem 3.2, p.14], there is a solution $x(t)$ of the equation

$$
-x^{\prime \prime}+\rho p(t) x=f(t, x),
$$

with the maximum existence interval $\left(\omega^{-}, 1\right]$ such that $x(1)=x_{0}, x^{\prime}(1)=$ $-(c / d) x_{0}$ and there is a subsequence of $\left\{x_{n}(t)\right\}$ - we denote it again by $\left\{x_{n}(t)\right\}-$ such that $\left\{x_{n}(t)\right\}$ and $\left.\left\{x_{n}^{\prime}(t)\right)\right\}$ converge uniformly to $x(t)$ and $x^{\prime}(t)$ on any compact subintervals of $\left(\omega^{-}, 1\right]$. Because $\bigcup_{n=1}^{\infty}\left[a_{n}, 1\right]=(0,1]$, and $\alpha(t) \leq x_{n}(t) \leq \beta(t), \quad t \in\left[a_{n}, 1\right]$, one can easily see that $\alpha(t) \leq x(t) \leq$ $\beta(t)$ for $t \in\left(\omega^{-}, 1\right]$. This leads additionally to the fact that $\left(\omega^{-}, 1\right]=(0,1]$, from the Extension Theorem. Also, $x(t)$ satisfies $x(0)=0$, because $\alpha(t)$ and $\beta(t)$ do, and $c x(1)+d x^{\prime}(1)=0$. Thus $x(t)$ is a $C^{1}(0,1]$ positive solution of problem (1.1).

This completes the proof of Theorem 3.2 for the case II): $b=0, d>0$.

The proof for the case III): $b>0, d=0$ is almost the same as for the case II). The proof of Theorem 3.2 is complete.

Acknowledgements. The authors would like to thank the referee for some valuable suggestions. We would like to thank Professor Li Sujie for his guidance and encouragement. 


\section{REFERENCES}

[1] D. O'Regan, Theory of Singular Boundary Value Problems, World Scientific Press, Singapore, 1994.

[2] — Existence Theory for Nonlinear Ordinary Differential Equations, Kluwer Academic Publishers, Dordrecht/Boston/London, 1997.

[3] S. D. Taliaferro, A nonlinear singular boundary value problem, Nonlinear Anal. TMA, 3 (1979), 897-904.

[4] Y. Zhang, Positive solutions of singular sublinear Emden-Fowler boundary value problems, J. Math. Anal. Appl., 185 (1994), 215-222.

[5] D. O'Regan D, Singular Dirichlet boundary value problems-I. Superlinear and nonresonance case, Nonlinear Analysis, TMA, 29(2) (1997), 221-245.

[6] Zhongli Wei, Positive solutions of singular boundary value problems of negative exponent Emden-Fowler equations, Acta Mathematica Sinica (in Chinese), 41(3) (1998), 655-662.

[7] _ Positive solutions of singular sublinear second order boundary value problems, Systems Science \& Mathematical Sciences, 11(1) (1998), 82-88.

[8] P. Hartman, Ordinary Differential Equations, 2nd Ed.,, Birkhauser, Boston, 1982.

\section{Zhongli Wei}

Department of Fundamental Courses

Shandong Institute of Architectural and Engineering

Jinan, Shandong, 250014

People's Republic of China

Changci Pang

Department of Fundamental Courses

Shandong Institute of Architectural and Engineering

Jinan, Shandong, 250014

People's Republic of China 